\title{
Two Water Stable Copper Metal-Organic Frameworks with Performance in the Electrocatalytic Activity for Water Oxidation
}

\author{
Xiuping Liu ${ }^{1}$, Yijun Wang ${ }^{2}$, Wenwen Liu ${ }^{1}$, Lianming Zhao ${ }^{1 *}$, and Wenyue Guo ${ }^{1, *}$ \\ ${ }^{1}$ College of Science, China University of Petroleum, Qingdao, Shandong 266580, People's Republic \\ of China \\ ${ }^{2}$ Linyi jun cheng leather co., LTD, Linyi, Shandong 276000, People's Republic of China
}

\begin{abstract}
Two novel water stable metal-organic frameworks, $\left[\mathrm{Cu}(\mathbf{L}) \cdot\left(4,4^{\prime} \text {-bipy }\right) \cdot\left(\mathrm{ClO}_{4}\right)\right]_{\mathrm{n}}(\mathbf{1}),\left[\mathrm{Cu}(\mathbf{L}) \cdot(\text { phen }) \cdot\left(\mathrm{ClO}_{4}\right) \cdot\left(\mathrm{H}_{2} \mathrm{O}\right)\right]_{2}(\mathbf{2})$, have been constructed by $\mathrm{HL}=[5-$ Mercapto-1-methyl] tetrazole acetic acid and $\mathrm{Cu}$ (II) salt in the presence of assistant N-containing ligands. MOF 1 and MOF 2 with open $\mathrm{Cu}^{\mathrm{II}}$ sites, resulting the framework $\mathbf{1}$ and $\mathbf{2}$ show electrocatalytic activity for water oxidation in alkaline solution. The electrochemical properties of complex for oxygen evolution reaction (OER) were evaluated by linear sweep voltammetry (LSV) and the Tafel slopes. Complex $\mathbf{1}$ has a higher LSV activity with a lower over potential of $1.54 \mathrm{~V}$ and a much higher increase in current density. Meanwhile, the Tafel slope of complex $1\left(122.0 \mathrm{mV} \mathrm{dec}^{-1}\right)$ is much lower than complex $2\left(243.5 \mathrm{mV} \mathrm{dec}^{-1}\right)$. This phenomenon makes complex $\mathbf{1}$ a promising porous material for electrocatalytic activity.
\end{abstract}

\section{Introduction}

Metal-organic frameworks (MOFs), as a new and vital class of porous crystalline materials, have received considerable attention in the past decades for their potential applications in the adsorption, catalysis, biology and others. ${ }^{[1]}$ The N-donor molecules and theirs derivatives have been widely used as the secondary ligands because of their rigid skeletons and the structures formed are more predictable. ${ }^{[2]}$ In the present paper, the ligand contains carboxylica acid and neutral four nitrogen thiazole ring.

Recently, some reports have shown that most of MOFs are unstable and lose their structural when exposed in water. Because the relatively weak metal-oxygen bonds within the frameworks are easily attacked by water molecules and lose their structures. ${ }^{[2]}$ Fortunately, the incorporation of hydrophobic functional groups within the frameworks might largely enhance the M-O bonds and thus improve the water resistance of the MOFs in an efficient manner. For example, introducing a $-\mathrm{CH}_{3}$ functional group within the framework might be enhance the water/moisture stability of the frameworks. ${ }^{[3]}$

Most of the reports on MOFs were focused on structures, gas-uptake, and so on, and the electrochemical reactivity of MOF systems, especially for the oxygen evolution reaction

\footnotetext{
*Corresponding author: lmzhao@upc.edu.cn
} 
(OER) was seldom studied. ${ }^{[4]}$ Therefore developing highly efficient electrocatalysts for OER has emerged as one of the research hot spots in clean energy generation and storage. ${ }^{[5]}$ It is of great importance to explore the series of materials to substitute traditional costeffective oxygen evolution catalysts. However, the well-established catalysts for water oxidation up to now are mainly recorded as some precious metals or traditional metal materials, ${ }^{[6]}$ and the MOFs used for OER are nearly unexplored. ${ }^{[7]}$ In this context, we present the syntheses, crystal structures and the OER properties of the two complexes, namely $\left[\mathrm{Cu}(\mathbf{L})\left(4,4^{\prime} \text {-bipy }\right)\right]_{\mathrm{n}}(\mathbf{1}),[\mathrm{Cu}(\mathbf{L})(\text { phen })]_{\mathrm{n}}(\mathbf{2})$, which incorporates $4,4^{\prime}$-bipy and phen ligands respectively.

\section{Experiment}

\subsection{Reagent and instruments}

All the reagents and solvents employed were commercially available and used as received without further purification except HL. X-ray powder diffractions were measured on a Panalytical X-Pert pro diffractometer with $\mathrm{Cu}-\mathrm{K} \alpha$ radiation. The elemental analyses $(\mathrm{C}, \mathrm{H}$, and $\mathrm{N}$ ) were determined on a CE instruments EA 1110 analyzer. TG curves were measured from 40 to $900{ }^{\circ} \mathrm{C}$ on a Mettler Toledo TGA instrument at a heating rate $10^{\circ} \mathrm{C} / \mathrm{min}$ under the $\mathrm{N}_{2}$ atmosphere $(100 \mathrm{~mL} / \mathrm{min})$.

\subsection{Synthesis of [5-Mercapto-1-methyl] tetrazole acetic acid}

The mixture of $1.0 \mathrm{~g}$ 5-Mercapto-1-methyltetrazole and the solution of $0.4 \mathrm{~g} \mathrm{NaOH}$ in $10 \mathrm{~mL}$ deionized water in a reaction flask at room temperature, the solution of $1.06 \mathrm{~g}$ sodium chloroacetate in $20 \mathrm{~mL}$ deionized water was added dropwise, then the mixture was stirred at $50{ }^{\circ} \mathrm{C}$ for $5 \mathrm{~h}$. After cooled, the reacting mixture was acidified by $1 \mathrm{~mol} / \mathrm{L}$ hydrochloric acid to $\mathrm{pH}=3.5$. In this process form a precipitation. The precipitation was filtered, and followed by ethyl rinsed, dried and finally became white powder, with a yield of $90 \%$, which was purified by recrystallization in ethanol-water to get white crystals.

\subsection{Synthesis of complex (1)}

Pale-blue block-shaped crystals of 1 can be easily synthesized from $\mathrm{Cu}\left(\mathrm{NO}_{3}\right)_{2}$ (2 mmol), 4,4'dithiosalicylic $(2 \mathrm{mmol})$ and $\mathrm{HL}(\mathrm{HL}=$ [5-Mercapto-1-methyl] tetrazole acetic acid in $10 \mathrm{ml}$ ethanol/water $(\mathrm{V} / \mathrm{V}=1: 1)$ solvents. And then were sealed in a pressure-resistant glass bottle and slowly heated to $75{ }^{\circ} \mathrm{C}$ from room temperature. After keeping at $75{ }^{\circ} \mathrm{C}$ for 3000 min, the mixture was slowly cooled to $30{ }^{\circ} \mathrm{C}$ at a rate of $7{ }^{\circ} \mathrm{C} / \mathrm{h}$. Blue rectangular block crystals suitable for single-crystal $\mathrm{X}$-ray diffraction were obtained. The products were washed with $\mathrm{H}_{2} \mathrm{O}$ and dried in air, $60 \%$ yield based on $\mathrm{Cu}^{\mathrm{II}}$. Elem anal. Calcd. for $\mathrm{C}_{14} \mathrm{H}_{13} \mathrm{ClCuN}_{6} \mathrm{O}_{6} \mathrm{~S}(\%)$ : C, 33.86; H, 2.17; N, 16.65; S, 6.46. Found (\%): C, 34.12; H, 2.18; $\mathrm{N}, 17.01 ; \mathrm{S}, 6.5$.

\subsection{Synthesis of complex (2)}

Metal organic framework 2 was synthesized in an analogous procedure to 1 except that 4,4' dithiosalicylic was replaced by phen, giving blue rectangular block X-ray-quality crystals in a $65 \%$ yield. Anal. Calcd. (\%) for $\mathrm{C}_{16} \mathrm{ClCuN}_{6} \mathrm{O}_{7} \mathrm{~S}(\%)$ : C, 36.94; H, 2.75; N, 16.16; S, 6.17 . Found (\%): C, 35.92; H, 2.77; N, 15.58; S, 5.70. The crystal data collection and refinement processes are summarized in Table 1. 
Table 1. Crystal data and structure parameters for the complexes $\mathbf{1}$ and $\mathbf{2}$.

\begin{tabular}{|c|c|c|}
\hline Complex & $\mathbf{1}$ & $\mathbf{2}$ \\
\hline Formula & $\mathrm{C}_{14} \mathrm{H}_{13} \mathrm{ClCuN}_{6} \mathrm{O}_{6} \mathrm{~S}$ & $\mathrm{C}_{16} \mathrm{ClCuN}_{6} \mathrm{O}_{7} \mathrm{~S}$ \\
\hline Formula weight & 492.15 & 519.27 \\
\hline Temperature/K & $293(2)$ & $293(2)$ \\
\hline Crystal system & triclinic & triclinic \\
\hline Space group & $\mathrm{I} / \mathrm{c}$ & $\mathrm{P}-1$ \\
\hline $\mathrm{a} / \AA$ & $19.524(4)$ & $10.5420(6)$ \\
\hline $\mathrm{b} / \AA$ & $11.14515(19)$ & $13.3290(14)$ \\
\hline $\mathrm{c} / \AA$ & $16.3628(3)$ & $87.056(9)$ \\
\hline$\alpha /\left(^{\circ}\right)$ & 90 & $76.136(8)$ \\
\hline$\beta /\left(^{\circ}\right)$ & $94.5919(17)$ & $72.575(8)$ \\
\hline$\left.\gamma /{ }^{\circ}\right)$ & 90 & $1011.51(18)$ \\
\hline $\mathrm{V} / \AA 3$ & $3620.19(12)$ & 2 \\
\hline $\mathrm{Z}$ & 8 & 1.519 \\
\hline $\mathrm{Dc} /(\mathrm{g} \cdot \mathrm{cm}-3)$ & 1.519 & 512.0 \\
\hline $\mathrm{F}(000)$ & 1992 & \\
\hline
\end{tabular}

\section{Results and discussions}

\subsection{Crystal structure of $\left[\mathrm{Cu}(\mathrm{L}) \cdot\left(4,4^{\prime}-\mathrm{bipy}^{\prime} \cdot\left(\mathrm{ClO}_{4}\right)\right]_{\mathrm{n}} 1\right.$}

Single-crystal X-ray diffraction analysis reveals that complex 1 possess 2D lamellar structure, crystallizing in monoclin I2/c space group. As shown in Fig. 1, The asymmetric unit in complex 1 contains one crystallographic independent $\mathrm{Cu}^{\mathrm{II}}$ ion, two $\mathbf{L}$ ligand, two 4,4'-bipy ligand, and one uncoordinated $\mathrm{ClO}_{4}$ anion. Complex $\mathbf{1}$ is a monocline laser crystal. Each $\mathrm{Cu}^{\mathrm{II}}$ atom is five-coordinated by two $\mathrm{O}$ atoms from two ligands and three $\mathrm{N}$ atoms from two 4,4'-bipy and one ligand. At last, the 2D infinite chains were further stacked into a three-dimensional supramolecular framework through $\pi-\pi$ stacking and weak interactions (Fig. 1a).

\subsection{Crystal structure of $\left[\mathrm{Cu}(\mathrm{L}) \cdot(\text { phen }) \cdot\left(\mathrm{ClO}_{4}\right) \cdot\left(\mathrm{H}_{2} \mathrm{O}\right)\right]_{2} 2$}

X-ray single-crystal diffraction reveals that complex 2 crystallized in monoclinic P-1 space group. The asymmetric unit in complex 2 contains one crystallographic independent $\mathrm{Cu}^{\mathrm{II}}$ ion, two lattice water molecular, two L ligand, two phen ligand, and one uncoordinated $\mathrm{ClO}_{4}$ anion. Each $\mathrm{Cu}^{\mathrm{II}}$ ion is five-coordinated by two $\mathrm{O}$ atoms from one ligands and water, three $\mathrm{N}$ atoms from one phen and one ligand, and the adjacent two $\mathrm{Cu}^{\mathrm{II}}$ centers are bridged by two ligand to form the framework (Fig. 1e). 

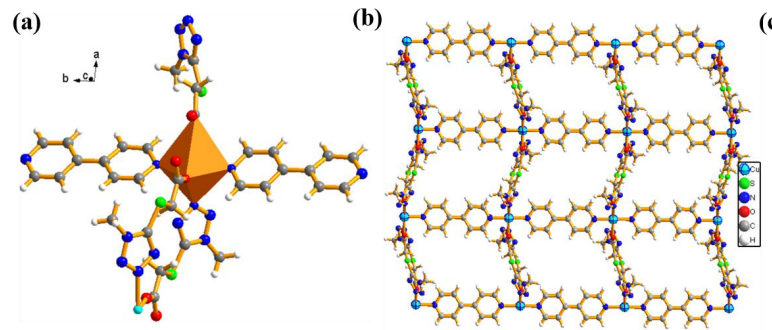

(c)

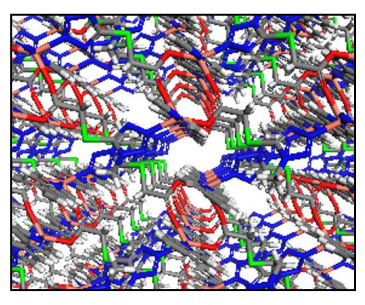

(d)

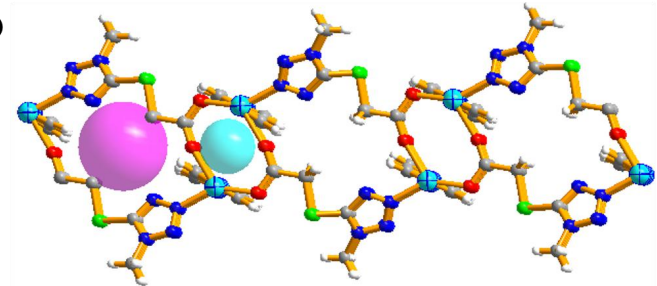

(e)

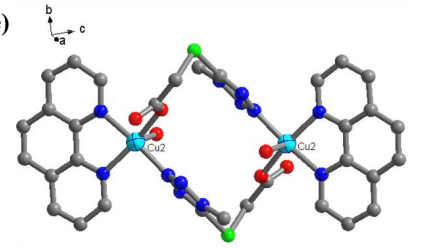

Fig. 1. (a) Coordination environment of $\mathrm{Cu}(\mathrm{II})$ center, (b) $2 \mathrm{D}$ sheet of $\mathbf{1}$, (c) A perspective view along the $\mathrm{c}$ axis showing the hexagonal shapedchannel in $\mathbf{1}$, (d) view of the $2 \mathrm{D}$ framework of 1 along the $\mathrm{c}$ axis showing the $1 \mathrm{D}$ open channels, (e) Coordination environment of $\mathrm{Cu}(\mathrm{II})$ center of $\mathbf{2}$.

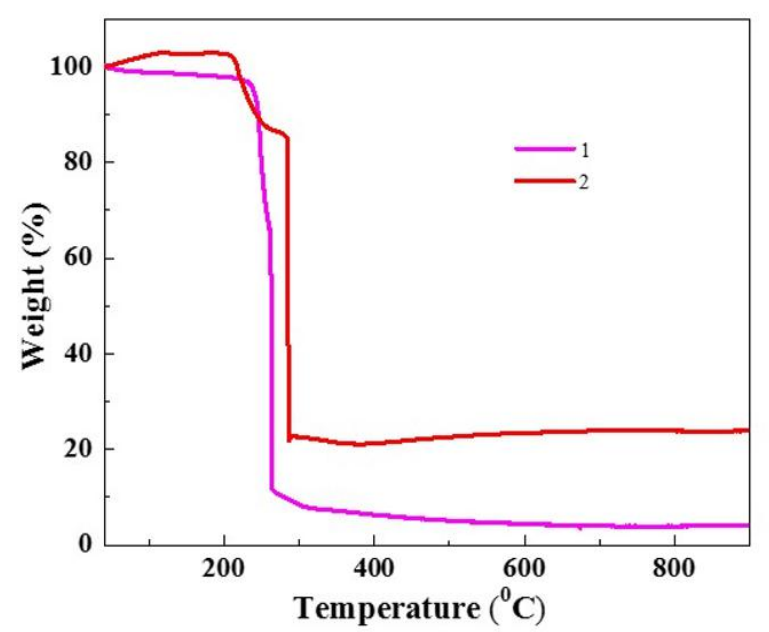

Fig. 2. TG curve of complex 1 and 2.

\subsection{Thermal analysis and powder X-ray diffraction}

The thermal behavior of sample 1 and sample 2 were studied by TGA under $\mathrm{N}_{2}$ atmosphere with a heating rate of $10{ }^{\circ} \mathrm{C} / \mathrm{min}$ (Fig. 2). For complex $\mathbf{1}$, up to $220{ }^{\circ} \mathrm{C}, \mathbf{1}$ begins to decompose. As for complex 2, above $270{ }^{\circ} \mathrm{C}$, complex 1 begins to decompose. This was further confirmed by the temperature-dependent PXRD results.

Variable-temperature PXRD for $\mathbf{1}$ and $\mathbf{2}$ were measured from room temperature to 200 ${ }^{\circ} \mathrm{C}$. As clearly exhibited in Fig. 3, as the temperature increases from 25 to $200{ }^{\circ} \mathrm{C}$, the PXRD pattern remains almost unchanged in terms of both the number and positions of the peaks, indicating the unchanged framework of $\mathbf{1}$ and $\mathbf{2}$ over this temperature range. This is also true for crystals of $\mathbf{1}$ and $\mathbf{2}$ upon treatment in boiling water or soaking in alcohol. Thus the stabilities of $\mathbf{1}$ and $\mathbf{2}$ in aqueous solutions were investigated by PXRD (Fig. 3). For these tests, $40 \mathrm{mg}$ of as-synthesized $\mathbf{1}$ and $\mathbf{2}$ were immersed in boiling water for an hour and soaked in aqueous solutions, methanol solutions for 2 days at room temperature. According 
to these results, indicating the maintain of the porous structures of complex $\mathbf{1}$ and $\mathbf{2}$ in boiling water and methanol solutions. This may be due to the hydrophobic functional groups $\left(-\mathrm{CH}_{3}\right)$ are close to the copper center within the framework of $\mathbf{1}$ and $\mathbf{2}$, and could prevent the $\mathrm{Cu}-\mathrm{O}$ and $\mathrm{Cu}-\mathrm{N}$ bonds from attacking by water and thus effectively enhance the hydrophobic properties. In addition, the experimental patterns are in good agreement with simulated patterns generated from the results of single-crystal diffraction data, demonstrating the phase purity of the product nicely.

(a)

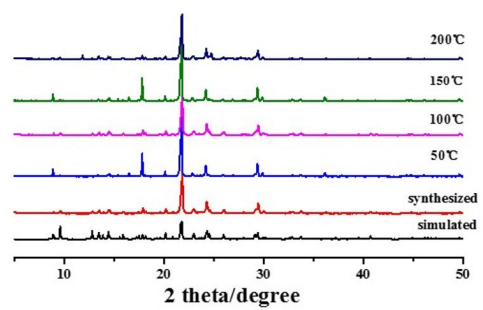

(c)

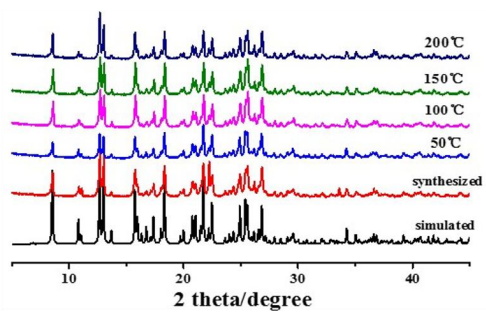

(b)

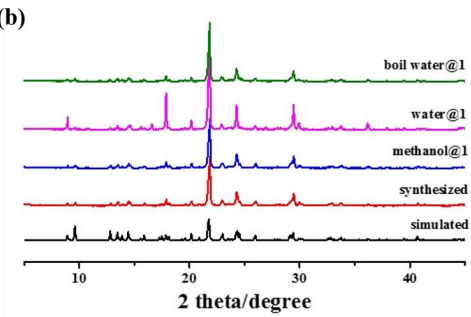

(d)

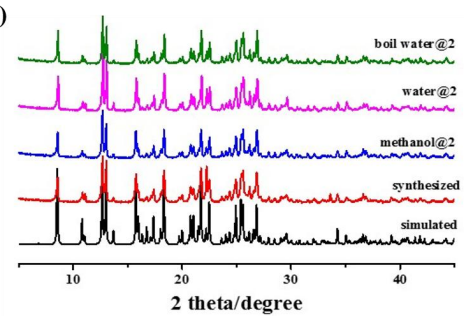

Fig. 3. PXRD patterns of 1 and (a) patterns from room temperature to $200{ }^{\circ} \mathrm{C}$; (b) patterns of samples with distinct chemical treatments. PXRD patterns of 2 (c) patterns from room temperature to $200{ }^{\circ} \mathrm{C}$; (d) patterns of samples with distinct chemical treatments.

\subsection{Electrochemistry activity}

Electrocatalytic properties for a myriad of electrochemical reactions such as OER have gained great research attention. ${ }^{[8]}$ In recent years, MOFs structures were seldom reported as OER catalyst. Therefore, we study the electrocatalytic activity of the complex $\mathbf{1}$ and complex 2. Electrochemical measurements were performed at an electrochemical workstation (Gamry Reference 600 Instruments, USA). The measurement was performed in $40 \mathrm{~mL}$ of $1.0 \mathrm{M} \mathrm{KOH} \mathrm{(aq)} \mathrm{electrolyte} \mathrm{prepared} \mathrm{using} 18 \mathrm{M} \Omega$ deionized water and purged with $\mathrm{O}_{2}$ before the measurement for half an hour in order to saturate the solution. The potentials reported in this paper were converted from vs. SCE to vs. RHE according to the equation $\mathrm{E}(\mathrm{RHE})=\mathrm{E}(\mathrm{SCE})+1.07 \mathrm{~V}$. Before measurement, the GCE was polished with $1.0,0.3$ and $0.05 \mu \mathrm{m}$ alumina powder. Then it was ultrasonicated in deionized water and the absolute ethanol for $10 \mathrm{~min}$ several times. For the preparation of the sample inks, $5 \mathrm{mg}$ of the as-prepared sample was dispersed in $1 \mathrm{~mL}$ dichloromethane solution and ultrasonicated for half an hour. Then, the slurry of $10 \mathrm{uL}$ was pipetted on the surface of GCE and dried in air for use. Linear sweep voltammetry (LSV) was conducted from $1.2 \mathrm{~V}$ to $1.85 \mathrm{~V}$ vs. RHE with a scan rate of $10 \mathrm{mV} \mathrm{s}^{-1}$. iR compensation was applied in it.

The electrocatalytic properties for OER of the two prepared samples are compared through the electrochemical measurement as is shown in Fig. 4. The linear sweep voltammetry (LSV) of the two samples are compared (Fig. 4a). It can be seen that the complex 1 has a higher LSV activity with a lower over potential of $1.54 \mathrm{~V}$ and a much higher increase in current density. The Tafel slopes of the two samples extracted from Fig. 1a are exhibited in Fig. 4b. The Tafel slope of complex $1\left(122.0 \mathrm{mV} \mathrm{dec}{ }^{-1}\right)$ is much lower 
than complex 2 (243.5 $\left.\mathrm{mV} \mathrm{dec}^{-1}\right)$, leading to an advantage for practical catalytic application. Thus, complex 1 has a much better electrocatalytic activity owing to the lower over potential and Tafel slope. This may be due to the follow reasons, first, it is known that the electrocatalytic reaction is primarily a surface phenomenon, ${ }^{[9]}$ and complex $\mathbf{1}$ has a high specific surface area and offers sufficient active sites for OER, which could be verified by the program Poreblazer V.3.0.2.51 and its structure; second, the ligand is supposed to promote electron transport at the heterogeneous surface due to its excellent electrical conductive properties, and it acts as an ideal platform for loading $\mathrm{Cu}^{\mathrm{II}}$; third, as carbon- and nitrogen-based organic frameworks have showed their promising electrocatalytic properties, such as in The hydrogen evolution reaction (HER), oxygen reduction reaction (ORR), and OER. ${ }^{[10]}$
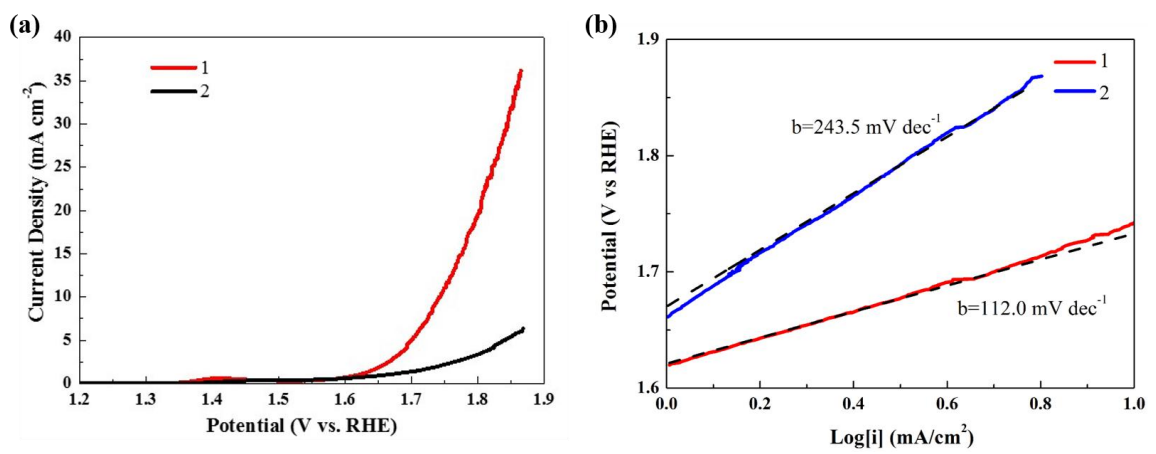

Fig. 4. Electrocatalytic activity of complex 1 and 2 for OER. (a) Polarization curves and (b) Tafel plots in $1.0 \mathrm{M} \mathrm{KOH}$ and $0.2 \mathrm{M}$ PBS solution $\left(10 \mathrm{mV} \mathrm{s}^{-1}\right)$.

\section{Summary}

In summary, two Cu-MOFs: $\left[\mathrm{Cu}(\mathbf{L}) \cdot(4,4 \text {-bipy }) \cdot\left(\mathrm{ClO}_{4}\right)\right]_{\mathrm{n}}(\mathbf{1}),\left[\mathrm{Cu}(\mathbf{L}) \cdot(\text { phen }) \cdot\left(\mathrm{ClO}_{4}\right) \cdot\left(\mathrm{H}_{2} \mathrm{O}\right)\right]_{2}$ (2), based on ligand $\mathrm{HL}$ and $\mathrm{Cu}\left(\mathrm{NO}_{3}\right)_{2}$ have been successfully obtained. The incorporation of hydrophobic functional groups (methyl) on the ligands attributes to the two new water stable $\mathrm{Cu}-\mathrm{MOF}$. Compared with those non-noblemetal electrocatalytic materials reported recently, the complex 1 electrode presents a large exchange current density and a relatively small Tafel slope. We suggesting that complex $\mathbf{1}$ is a promising low-cost and earthabundant metallic electrocatalyst for OER.

\section{References}

1. X.P. Liu, Z.Y. Xiao, J. Xu, W.B. Xu, P.P. Sang, L.M. Zhao, H.Y. Zhu, D.F. Sun, W.Y. Guo, J. Mater. Chem. A 4, 13844 (2016)

2. K. Tan, N. Nijem, Y. Gao, S. Zuluaga, J. Li, T. Thonhauser, Y.J. Chabal, CrystEngComm 17, 247 (2015)

3. M.E. Braun, C.D. Steffek, J Kim, P.G. Rasmussen, O.M. Yaghi, Chem. Commun. 24, 2532 (2001)

4. K. Sakaushi, M. Antonietti, Acc. Chem. Res. 48, 1591 (2015)

5. C.L. Tan, H. Zhang, Chem. Soc. Rev. 44, 2713 (2015)

6. S.S. Wang, G.Y. Yang, Chem. Rev. 115, 4893 (2015)

7. F. Schüth, Chem. Mater. 26, 423 (2014) 
8. J.S. Li, S.L. Li, Y.J. Tang, M. Han, Z.H. Dai, J.C. Bao and Y.Q. Lan, Chem. Commun. 51, 2710 (2015)

9. J. Xiao, Q. Kuang, S.H. Yang, F. Xiao, S. Wang and L. Guo, Sci. Rep, 3, 2300 (2013)

10. Y. Cheng, J. Zhang and S. P. Jiang, Chem. Commun. 51, 13764 (2015) 